PROCEEDINGS OF THE

AMERICAN MATHEMATICAL SOCIETY

Volume 133, Number 5, Pages 1491-1495

S 0002-9939(04)07650-6

Article electronically published on November 1, 2004

\title{
COVERINGS BY CONVEX BODIES AND INSCRIBED BALLS
}

\author{
VLADIMIR KADETS
}

(Communicated by N. Tomczak-Jaegermann)

\begin{abstract}
Let $H$ be a Hilbert space. For a closed convex body $A$ denote by $r(A)$ the supremum of the radiuses of balls contained in $A$. We prove that $\sum_{n=1}^{\infty} r\left(A_{n}\right) \geq r(A)$ for every covering of a convex closed body $A \subset H$ by a sequence of convex closed bodies $A_{n}, n \in \mathbb{N}$. It looks like this fact is new even for triangles in a 2-dimensional space.
\end{abstract}

\section{INTRODUCTION}

Recall that by a plank of width $w$ in a Hilbert space one means a set of the form

$$
P=\left\{h \in H:\left|\left\langle h-h_{0}, e\right\rangle\right| \leq \frac{w}{2}\right\},
$$

where $\|e\|=1$. According to T. Bang's theorem [3], if a sequence $P_{n}$ of planks of widths $w_{n}$ covers a ball of diameter $w$, then $\sum w_{n} \geq w$. (To be more precise this is a particular case of Bang's theorem. The complete statement includes a convex set $P$ of minimal width $w$ instead of a ball.)

K. Ball generalized Bang's theorem to coverings of a ball in a Banach space, for planks defined with the help of linear functionals instead of inner product.

Our work is inspired by Bang's and Ball's theorems. The idea was to find a "symmetric" generalization of these theorems, where the elements of the covering and the covered set are of the same nature, and they are measured "from inside". In this paper we prove such a generalization for sets in a Hilbert space. An analogous question for general Banach spaces remains open. In the proof we use ideas from Ball's exposition of Bang's theorem.

\section{The MAIN RESUlT}

For simplicity throughout the paper we consider real Hilbert spaces (finite- or infinite-dimensional). All the results are true for complex spaces too: the only thing which one must change in the proofs is the equation of a real hyperplane: in the complex case such an equation uses not the inner product itself, but the real part of the inner product.

Received by the editors November 6, 2003 and, in revised form, January 7, 2004.

2000 Mathematics Subject Classification. Primary 52A37; Secondary 46C05.

Key words and phrases. Hilbert space, convex sets, inscribed ball.

The author expresses thanks to the Department of Mathematics, University of MissouriColumbia, and especially to Professor Nigel Kalton for hospitality and a fruitful working atmosphere. 
Let $H$ be a Hilbert space, and let $B$ and $S$ be its unit ball and its unit sphere respectively. By "body" in $H$ we mean a closed subset with non-empty interior. For a convex body $A$ denote by $r(A)$ the supremum of the radiuses of balls $x+a B$ contained in $A$.

If $A$ is a ball of radius $r$, then $r(A)=r$. If $A$ is a plank of width $h$, then $r(A)=\frac{h}{2}$.

Theorem 2.1. If a convex body $A \subset H$ is covered by a sequence of convex bodies $A_{n}, n \in \mathbb{N}$, then $\sum_{n=1}^{\infty} r\left(A_{n}\right) \geq r(A)$.

To prove the theorem we need first some lemmas. The goal of the lemmas is to prove that a convex bounded body in a Hilbert space can be approximated in some sense by a polytope with a finite number of faces.

Lemma 2.2. Let $A \subset H$ be a bounded convex body. Then there is a ball $U \subset A$ with $r(U)=r(A)$.

Proof. Denote $r(A)=r$. For every $p \in[0, r)$ consider the set $A_{p}$, consisting of all those $x \in A$ for which $x+p B \subset A$. Each $A_{p}$ is a bounded convex closed set, and hence it is a weak compact set. Since $A_{p} \neq \emptyset$ and decreases as $p \rightarrow r$, there is a point $o_{1} \in \bigcap_{p \in[0, r)} A_{p}$. Then $U=o_{1}+r B$ will be the ball we need.

Lemma 2.3. Let $A \subset H$ be a bounded convex body, $r(A)=r$, and let $U=o_{1}+r B$ be the ball from the previous lemma. Then for every $\varepsilon>0$ there is a finite subset $G=\left\{g_{1}, \ldots, g_{n}\right\} \subset r B$ such that $(1+\varepsilon) g_{i}+o_{1} \in H \backslash A$ and

$$
\operatorname{dist}\left(\operatorname{conv}\left\{g_{i}\right\}_{i=1}^{n}, 0\right) \leq \varepsilon .
$$

Proof. Without loss of generality, we may assume $o_{1}=0$ (otherwise shift the picture). We must prove that the closed convex hull of $(1+\varepsilon) r B \backslash A$ contains 0 .

Assume it is not so. By the Hahn-Banach theorem, there is a hyperplane $P$, strictly separating $(1+\varepsilon) r B \backslash A$ from 0 . This means that the set $F$ - the bigger part of the ball $(1+\varepsilon) r B$, lying on the same side of $P$ as 0 , is included in $A$. The subset $F \cup r B$ of $A$ evidently contains a ball of radius bigger than $r$, which contradicts the definition of $r$.

Lemma 2.4. Let $x, y \in H,\|x\|=1+\varepsilon$, and suppose the hyperplane

$$
\left\{h \in H:\langle h, y\rangle=\|y\|^{2}\right\}
$$

generated by $y$ separates $x$ from the unit ball. Then $\|x-y\| \leq \delta(\varepsilon)$, where $\delta(\varepsilon)$ tends to 0 as $\varepsilon$ tends to 0.

Proof. Our conditions mean that

$$
\langle x, y\rangle \geq\|y\|^{2}
$$

and

$$
\|y\|=\sup _{h \in B}\langle h, y\rangle \leq\|y\|^{2}
$$

i.e. $\|y\| \geq 1$. We have

$$
\|x-y\|^{2}=\|x\|^{2}-\|y\|^{2}+2\left(\|y\|^{2}-\langle x, y\rangle\right) \leq\|x\|^{2}-\|y\|^{2} \leq(1+\varepsilon)^{2}-1 .
$$


Lemma 2.5. Let $A \subset H$ be a bounded convex body, $r(A)=r$, and let $U=o_{1}+r B$ be the ball from Lemma 2.2. Then for every $\varepsilon>0$ there is a finite subset $V=$ $\left\{v_{1}, \ldots, v_{n}\right\} \subset r B$ ( $n$ depends on $\varepsilon$ ) such that

$$
A \subset W:=\bigcap_{v \in V}\left\{h \in H:\left\langle h-o_{1}, v\right\rangle<r^{2}+\varepsilon\right\} \text {, and }
$$

$$
\operatorname{dist}(\operatorname{conv} V, 0) \leq \varepsilon
$$

Proof. Apply Lemma 2.3 for a small $\varepsilon_{1}$ to obtain the corresponding $G=\left\{g_{1}, \ldots, g_{n}\right\}$ $\subset r B$. Since $\left(1+\varepsilon_{1}\right) g_{i}+o_{1} \in H \backslash A$, one can separate $\left(1+\varepsilon_{1}\right) g_{i}+o_{1}$ from $A$ by a hyperplane

$$
P_{i}=\left\{h \in H:\left\langle h-o_{1}, w_{i}\right\rangle=\left\|w_{i}\right\|^{2}\right\} .
$$

Since $U=o_{1}+r B \subset A, P_{i}$ separates $U$ from $\left(1+\varepsilon_{1}\right) g_{i}+o_{1}$. This means that the hyperplane

$$
\left\{h \in H:\left\langle h, w_{i}\right\rangle=\left\|w_{i}\right\|^{2}\right\}
$$

separates $r B$ from $\left(1+\varepsilon_{1}\right) g_{i}$, and by Lemma 2.4 $w_{i}$ is "very close" to $g_{i}$, and $\left\|w_{i}\right\|$ is "very close" to $r$. This means in turn that when $\varepsilon_{1}$ is small enough, the elements $v_{k}=r \frac{w_{k}}{\left\|w_{k}\right\|}, k=1,2, \ldots, n$ fulfill the demands of this lemma.

Lemma 2.6. For a convex body $A \subset B$ and for arbitrary $\delta>0$ there is a polytope $W$ of the form

$$
\bigcap_{k=1}^{n}\left\{h \in H:\left\langle h-o_{1}, v_{k}\right\rangle<a_{k}\right\},
$$

such that $W \supset A$ and $r(W \cap B) \leq r(A)+\delta$.

Proof. The polytope $W$ can be taken from (2) of the previous Lemma 2.5 with $\varepsilon$ small enough. Let us show this. Consider $U=o_{1}+r B$ from Lemma 2.2, For simplicity assume $o_{1}=0$ (the general case differs not too much from this one). Assume, contrary to our statement, that $W \cap B$ contains a ball of the form $U_{0}=$ $o_{0}+(r+\delta) B$ (and automatically $\left.\left\|o_{0}\right\| \leq 1\right)$. Then $W \supset \operatorname{conv}\left\{U_{0}, U\right\}$. According to condition (3), since $(1+\varepsilon) V$ lies outside $W$,

$$
\text { dist }(\operatorname{conv}((1+\varepsilon) U \backslash W), 0) \leq(1+\varepsilon) \varepsilon,
$$

and hence

$$
\operatorname{dist}\left(\operatorname{conv}\left((1+\varepsilon) U \backslash \operatorname{conv}\left\{U_{0}, U\right\}\right), 0\right) \leq(1+\varepsilon) \varepsilon .
$$

But for fixed $\delta$ and $\varepsilon \rightarrow 0$ the last inequality cannot be true, since the radius of $U_{0}$ equals $(r+\delta)$ and the distance between the centers of $U_{0}$ and $U$ is bounded by a number, independent of $\varepsilon$.

Proof of Theorem 2.1. Denote $r\left(A_{n}\right)=r_{n}$. By the definition of $r(A)$ we must prove that $\sum_{n=1}^{\infty} r_{n} \geq r(U)$ for every ball $U \subset A$. By homogeneity this means that it is sufficient to prove the theorem for the case of $A$ being the unit ball $B$ of $H$. Also without loss of generality one may assume $A_{n} \subset B$ : otherwise consider $A_{n} \cap B$ instead of $A_{n}$.

So the theorem can be reformulated as follows:

Let $\sum_{n=1}^{\infty} r_{n}<1$, and let $A_{n} \subset B$ be convex bodies with $r\left(A_{n}\right)=r_{n}$. Then

$$
B \backslash \bigcup_{n=1}^{\infty} A_{n} \neq \emptyset \text {. }
$$


According to Lemma 2.6 every $A_{n}$ may be included in the interior of a polytope (say, $C_{n}$ ), in such a way that the condition $\sum_{n=1}^{\infty} r\left(C_{n} \cap B\right)<1$ still takes place. So, in fact, we may assume that the interiors of all of the $A_{n}$ are relatively weakly open in $B$, and it is enough to prove that $B$ cannot be covered by the interiors of the $A_{n}$. But in this case, since $B$ is a weak compact set, it is sufficient to prove that $B$ cannot be covered by the union of finitely many interiors of the $A_{n}$. So we reduced our theorem to the case when the number of sets is finite (say, equals $N$ ).

Let us fix a sequence of positive $\delta_{n}$ with

$$
\sum_{n=1}^{N}\left(1+\delta_{n}\right) r_{n}<1
$$

and select $\varepsilon_{n}>0$ with

$$
\left(1+\delta_{n}\right)\left(r_{n}^{2}-6 N \varepsilon_{n}\right) \geq r_{n}^{2}+\varepsilon_{n} .
$$

For every $A_{n}$ apply Lemma2.5 with $\varepsilon=\varepsilon_{n}$ to get corresponding vectors $o_{n} \in A_{n}$, finite sets $V_{n} \subset r_{n} B$ and open polytopes

$$
W_{n}:=\bigcap_{v \in V_{n}}\left\{h \in H:\left\langle h-o_{n}, v\right\rangle<r_{n}^{2}+\varepsilon_{n}\right\},
$$

such that $A_{n} \subset W_{n}$ and

$$
\operatorname{dist}\left(\operatorname{conv} V_{n}, 0\right) \leq \varepsilon_{n}
$$

We are going to prove that

$$
B \backslash \bigcup_{n=1}^{N} W_{n} \neq \emptyset .
$$

Let us introduce an auxiliary space $X=H \oplus H_{1} \oplus H_{2} \oplus \ldots \oplus H_{N}$, the Hilbertian orthogonal direct sum of isometric copies of the original space $H$. Let $U_{n}: H \rightarrow H_{n}$ be the corresponding isometries.

Consider $K=V_{1} \times V_{2} \times \ldots \times V_{N}$, and for every $\bar{g}=\left(g_{1}, g_{2}, \ldots, g_{N}\right) \in K$ introduce

$$
f(\bar{g})=\sum_{n=1}^{N}\left(\left(1+\delta_{n}\right)\left(g_{n}+U_{n} g_{n}\right)-U_{n} o_{n}\right) .
$$

Fix $\bar{x}=\left(x_{1}, x_{2}, \ldots, x_{N}\right) \in K$ for which $\|f(\bar{x})\|$ is maximal. We are going to prove that

$$
x=\sum_{n=1}^{N}\left(1+\delta_{n}\right) x_{n}
$$

is the element we need: namely, $x$ belongs $B$ but does not belong to any of $W_{j}$.

First of all, due to (4), $\left\|\sum_{n=1}^{N}\left(1+\delta_{n}\right) x_{n}\right\| \leq \sum_{n=1}^{N}\left(1+\delta_{n}\right) r_{n}<1$, i.e. $x \in B$. Now for a fixed $j \in\{1,2, \ldots, N\}$ consider

$$
y_{j}=f(\bar{x})-\left(1+\delta_{j}\right)\left(x_{j}+U_{j} x_{j}\right) .
$$

Taking into account that, according to our construction,

$$
\left\|y_{j}+\left(1+\delta_{j}\right)\left(x_{j}+U_{j} x_{j}\right)\right\|=\|f(\bar{x})\| \geq\left\|y_{j}+\left(1+\delta_{j}\right)\left(v+U_{j} v\right)\right\|
$$

for all $v \in V_{j}$, we obtain for all $v \in V_{j}$,

$$
\left\langle y_{j},\left(1+\delta_{j}\right)\left(x_{j}+U_{j} x_{j}\right)\right\rangle \geq\left\langle y_{j},\left(1+\delta_{j}\right)\left(v+U_{j} v\right)\right\rangle .
$$


Due to condition (6) , there is a convex combination $\sum_{v \in V_{j}} \alpha_{v} v$, having norm less then $\varepsilon_{j}$. Multiplying (8) by $\alpha_{v}$ and adding for all $v \in V_{j}$ we obtain

$$
\left\langle y_{j},\left(1+\delta_{j}\right)\left(x_{j}+U_{j} x_{j}\right)\right\rangle \geq-2 \varepsilon_{j}\left(1+\delta_{j}\right)\left\|y_{j}\right\| \geq-6 N \varepsilon_{j}\left(1+\delta_{j}\right) .
$$

Let us transform the last inequality using the definition of $y_{j}$ and the pairwise orthogonality of the subspaces $H, H_{1}, \ldots, H_{N}$ :

$$
\begin{gathered}
\left\langle x-\left(1+\delta_{j}\right) x_{j}+\left(1+\delta_{j}\right) \sum_{n \neq j} U_{n} x_{n}-\sum_{n=1}^{N} U_{n} o_{n},\left(1+\delta_{j}\right)\left(x_{j}+U_{j} x_{j}\right)\right\rangle \\
\geq-6 N \varepsilon_{j}\left(1+\delta_{j}\right), \\
\left(1+\delta_{j}\right)\left\langle x, x_{j}\right\rangle-\left(1+\delta_{j}\right)^{2} r_{j}^{2}-\left(1+\delta_{j}\right)\left\langle U_{j} o_{j}, U_{j} x_{j}\right\rangle \geq-6 N \varepsilon_{j}\left(1+\delta_{j}\right), \\
\left(1+\delta_{j}\right)\left\langle x, x_{j}\right\rangle-\left(1+\delta_{j}\right)\left\langle o_{j}, x_{j}\right\rangle \geq\left(1+\delta_{j}\right)^{2} r_{j}^{2}-6 N \varepsilon_{j}\left(1+\delta_{j}\right) .
\end{gathered}
$$

By (5) we have

$$
\left\langle x-o_{j}, x_{j}\right\rangle \geq r_{j}^{2}+\varepsilon_{j},
$$

and this by definition of $W_{j}$ means that $x$ does not belong to $W_{j}$.

\section{REFERENCES}

[1] Keith Ball, Convex Geometry and Functional Analysis, in W.B.Johnson and J.Lindenstrauss (editors) Handbook of the geometry of Banach spaces, vol. 1 (2001), 161 - 194. MR 1863692 (2003c:52001)

[2] Keith Ball, The plank problem for symmetric bodies, Invent. Math. 104 (1991), 535 - 543. MR,1106748(92c:52003)

[3] T. Bang, A solution of the "Plank problem", Proc. Amer. Math. Soc. 2 (1951), 990 - 993. MR.0046672(13:769a)

Department of Mechanics and Mathematics, Kharkov National University, Pl. SvoBODY 4, 61077 KhaRKov, UKRAINE

E-mail address: vova1kadets@yahoo.com

Current address: Department of Mathematics, University of Missouri, Columbia, Missouri 65211 